\title{
Preface: Special Session SpS12 Modern views of the interstellar medium
}

The interstellar medium (ISM) plays an important role in the formation and evolution of a galaxy. The ISM provides the material to form stars and stars in turn inject radiation, metals, and mechanical energy into the ISM, altering the physical conditions, abundances, and distribution of the ISM and affecting future generations of star formation. It is thus essential that we understand the physical structure of the ISM, the physical processes that operate in the ISM, and the interplay between stars and ISM.

The physical structure and processes of the ISM are best studied in the Galaxy and nearby galaxies. Many large-scale surveys for different components of the ISM are available: HI - the International Galactic Plane Survey (IGPS) and The HI Nearby Galaxy Survey (THINGS) maps out the HI in nearby galaxies; dust - Spitzer, Herschel and Planck surveys of the Galactic plane and the Magellanic Clouds, Bolocam Galactic Plane Survey (BGPS), and the APEX Telescope Large Area Survey of the Galaxy (ATLASGAL); HII - the Wisconsin H-Alpha Mapper (WHAM) survey of the distribution and velocities of warm ionized gas in the Galaxy; $10^{6} \mathrm{~K}$ gas - Chandra and XMM-Newton observations; cosmic rays - Fermi Gamma-Ray Observatory's whole sky survey.

In the meantime, considerable progress has been made in the numerical modeling of local and global conditions of the ISM, its morphology, and its time-dependent evolution, owing to rapid advances in the development of hard- and software. It is now possible to follow the full non-linear evolution of a plasma by solving the hydro- or MHD equations in high resolution simulations with adaptive mesh refinement. One of the key results of the past years was to recognize and quantitatively describe the role of compressible turbulence in the ISM and its impact on the distribution of gas into phases, on the mixing of chemically enriched material, on the volume and mass filling factors of the ISM plasma, and on its heating and cooling history.

Special Session 12, "Modern Views of the ISM", is organized to update people on recent advances in the ISM observations and theories. The key topics include (1) physical structure and phase distribution of the ISM in a galaxy, (2) multi-wavelength observations of ISM in the Galaxy and nearby galaxies, (3) recent theory/MHD simulation of ISM in a galaxy, (4) interstellar disk-halo connection in galaxies, and (5) interplay between stars and ISM: star formation and feedback. This special session is dedicated to the late Professor John Dyson (1941-2010), the IAU Div VI president in 2003-2006, who pioneered the dynamical interactions of stellar winds and outflows with the interstellar gas.

\section{Scientific Organizing Committee}

D. Breitschwerdt (co-Chair, Germany), Y.-H. Chu (co-Chair, USA), M. de Avillez (Portugal), E. de Blok (South Africa), E. de Gouveia Dal Pino (Brazil), R.-J. Dettmar (Germany), E. Falgarone (France), T. Hartquist (UK), B.-C. Koo (Korea), N. McClureGriffiths (Australia), E. Ostriker (USA), J. X. Prochaska (USA), L. V. Tóth (Hungary), E. Vazquez-Semadeni (Mexico), K. Wada (Japan), M. Wolfire (USA), J. Yang (China).

You-Hua Chu (ed.)

Astronomy Department, University of Illinois, Urbana, IL 61801, USA; yhchu@illinois.edu 